\title{
Impact on Antijamming Performance of Channel Mismatch in GNSS Antenna Arrays Receivers
}

\author{
Zukun Lu, Junwei Nie, Feiqiang Chen, and Gang Ou \\ College of Electronic Science and Engineering, National University of Defense Technology, Changsha 410073, China \\ Correspondence should be addressed to Zukun Lu; luzukun@nudt.edu.cn
}

Received 28 June 2016; Accepted 20 November 2016

Academic Editor: Antonio Faraone

Copyright (C) 2016 Zukun Lu et al. This is an open access article distributed under the Creative Commons Attribution License, which permits unrestricted use, distribution, and reproduction in any medium, provided the original work is properly cited.

\begin{abstract}
The performance of antijamming is limited by channel mismatch in global navigation satellite system (GNSS) antenna arrays receivers. Only when the amplitude and phase characteristics of each array channels are the same is the interference likely to be completely suppressed. This paper analyzes the impact on antijamming performance of channel mismatch. We built the model of channel mismatch and derived the impact on transfer function with space-time adaptive processor (STAP) of channel mismatch in theory. The impact factor of channel mismatch is proposed by the fuzzy transfer function, which could directly reflect the antijamming performance under channel mismatch. In addition, every characteristic in the channel mismatch model is analyzed. The analysis results show that the greatest impacts on antijamming performance are the range of amplitude wave and the group delay bias, while the influence of the number of amplitudes is next. As for the effect of group delay fluctuation is the smallest.
\end{abstract}

\section{Introduction}

Since 1970s, global navigation satellite system (GNSS), whose representative is global position system (GPS), has a rapid development. GNSS has brought great convenience to people in traffic, and it is also widely used in military field [1]. The distance between earth surface and navigation satellite is about $27,000 \mathrm{~km}$. The navigation signal is very weak when it reaches the ground. The navigation receivers get the signals with the signal/noise ratio (SNR) is about $-30 \mathrm{~dB}$, which makes receivers vulnerable to all kinds of intentional or unintentional interference. The performance of antijamming has become a key indicator in military and some civilian applications [2,3]. According to the number of antennas, the antijamming technology of receivers can be divided into single antenna and antenna arrays, in which the single antenna could only suppress narrowband interference and the antenna arrays could suppress both narrowband and wideband interference [4]. Under the condition of navigation war, the receivers would face the strong wideband interference. The antenna arrays equipped with space-time adaptive processor (STAP) would be an optimal choice in the electromagnetic countermeasure [5]. The fundamental principle of STAP is array weight in digital baseband, which could suppress interference effectively when the amplitude and phase characteristics of each channel are unanimous [6]. However, each array channel is composed of the low noise amplifier (LNA), analog filter, inverter, and other analog devices. The manufacturing errors and environmental and other factors could lead to inconsistencies of amplitude and phase in each channel, which would cause channel mismatch $[7,8]$.

Channel mismatch has attracted the attention of scholars. The impact of channel mismatch on array gain is studied by space-only processor (SOP) in [9], which notes that the amplitude and phase mismatch would affect the performance of interference cancellation, which would lead to the degradation of antijamming performance. Literature [10] gives a preliminary analysis to the impact of channel mismatch on antenna arrays equipped with STAP. The estimated carrier phase would be biased due to the channel mismatch, and the greater mismatch would bring greater deviation. A correction method of channel mismatch based on the correction table is proposed in [11], which needs to measure the frequency response in all directions and estimate the bias of pseudocode and carrier phase which is introduced by antenna arrays, and it also needs to make the correction table bind to the 
receivers, whereas the nonideal characteristic of the channel might be changed in some time, whose method lacks certain robustness.

This paper further analyzes the impact of channel mismatch on antijamming performance with antenna arrays. First of all, we establish a model of channel mismatch and deduce the impact of channel mismatch on transfer function with STAP in theory. Then, the impact on antijamming performance is analyzed. We propose a concept of channel mismatch impact factor and point out the largest impact characteristic of channel mismatch to antijamming performance. Finally, simulation experiments are implemented to validate the correctness of the analytical results.

\section{Channel Mismatch Model}

Nonideal characteristics of the channel are mainly reflected in the nonflatness of the amplitude and nonlinearity of the phase. The impact of the interference suppression in antenna arrays is not the absolute value of each channel, but the different between each channel, which can be called channel mismatch [12].

Suppose the transfer function in the channel $n$ is $H_{n}(\omega)$, and literature [13] gives a channel mismatch model which can be written as

$$
H_{n}(\omega)= \begin{cases}A(\omega) e^{j \varphi(\omega)} & |\omega| \leq \pi B \\ 0 & \text { else, }\end{cases}
$$

where $B$ is the bandwidth of receivers. $A(\omega)$ and $\varphi(\omega)$ are the response of amplitude-frequency and phase-frequency, respectively. In the ideal case, $A(\omega)$ and $\varphi(\omega)$ satisfy the following relationship:

$$
\begin{aligned}
& A(\omega) \equiv 1 \\
& \varphi(\omega) \equiv 0 .
\end{aligned}
$$

A more accurate channel mismatch model has been demonstrated in [14] which can be expressed as

$$
\begin{aligned}
& H_{n}(\omega) \\
& \quad= \begin{cases}\left(1+R_{a} \cos \omega R_{a n}\right) e^{j\left(R_{p} \cos \omega R_{p n}+\omega R_{b}\right)} & |\omega| \leq \pi B \\
0 & \text { else, }\end{cases}
\end{aligned}
$$

where $R_{a}$ and $R_{p}$ are the range of amplitude and group delay wave, respectively. $R_{a n}$ and $R_{p n}$ are the number of amplitude and group delay waves, separately. $R_{b}$ is the group delay bias.

As for the antenna arrays with $N$ elements, the channel mismatch model can be written as

$$
\mathbf{H}(\omega)=\left[\begin{array}{llll}
H_{1}(\omega) & \cdots & H_{N}(\omega)
\end{array}\right]^{\mathrm{T}} .
$$

\section{Impact on Transfer Function}

STAP has obvious advantages compared to the simple time domain, frequency domain, and SOP. STAP could suppress a variety of interferences. Without increasing the antenna elements, the degree of freedom (DoF) of interference suppression can greatly increase, and its interference suppression capability has a quality improvement [15]. In addition, STAP could mitigate the impact of channel mismatch to interference suppression. The taps in STAP can compensate the channel mismatch.

The STAP equipped with $N$ elements and $M$ taps is shown in Figure 1 [16].

According to the STAP model shown in Figure 1, the received data in different sampling time could form a new data vector which could be expressed as

$$
\mathbf{x}=\left[\begin{array}{lllll}
\mathbf{X}^{\mathrm{T}}(t) & \mathbf{X}^{\mathrm{T}}(t-T) & \cdots & \mathbf{X}^{\mathrm{T}}(t-(M-1) T)
\end{array}\right]^{\mathrm{T}},
$$

where $\mathbf{X}(t)$ is the received data vector at the time of $t$.

Array weightings could be written as

$$
w=\left[w_{11}, \ldots, w_{N 1}, \ldots, w_{1 M}, \ldots, w_{N M}\right]^{\mathrm{T}}
$$

In order to conveniently perform analysis, assume the number of interferences is one and its power spectral density (PSD) is smooth. In addition, STAP is equipped with 2 elements and 2 taps.

The steering vector of time and space domain can be, respectively, denoted as

$$
\begin{aligned}
& v^{t}(\omega)=\left[\begin{array}{lll}
1 & \cdots & e^{-j \omega(M-1) T}
\end{array}\right]^{\mathrm{T}} \\
& v^{s}(\theta)=\left[\begin{array}{lll}
1 & \cdots & e^{-j \omega_{R}(N-1) \tau(\theta)}
\end{array}\right]^{\mathrm{T}},
\end{aligned}
$$

where $T$ is the sampling period, $\omega_{R}$ is the radio frequency (RF), and $\tau(\theta)$ is the delay of the two adjacent antenna elements and the signal or interference direction is $\theta$ and it can be further expressed as [17]

$$
\tau(\theta)=\frac{d \sin \theta}{c}
$$

where $d$ is the distance of the adjacent antenna elements with half wavelength and $c$ is the propagation speed of signal or interference.

According to (7), the steering vector of space-time domain can be written as

$$
\mathbf{a}(\omega, \theta)=v^{t}(\omega) \otimes v^{s}\left(\omega_{R}, \theta\right) .
$$

Suppose channel 1 is desirable, and channel 2 represents the nonideal characteristics, and its transfer function is $H$ that is related to the frequency with $\omega$. According to (4), the model of channel mismatch could be expressed as

$$
\mathbf{H}(\omega)=\left[\begin{array}{ll}
H_{0} & H
\end{array}\right]^{\mathrm{T}} .
$$

As for all frequency, $H_{0}$ in the above equation would satisfy the following relation:

$$
H_{0} \equiv 1 \text {. }
$$

Then, the steering vector of space-time domain could be improved as

$$
\mathbf{a}(\omega, \theta)=v^{t}(\omega) \otimes\left[v^{s}\left(\omega_{R}, \theta\right) \odot \mathbf{H}(\omega)\right],
$$

where $\otimes$ stands for Kronecker product and $\odot$ indicates Hadamard product. 


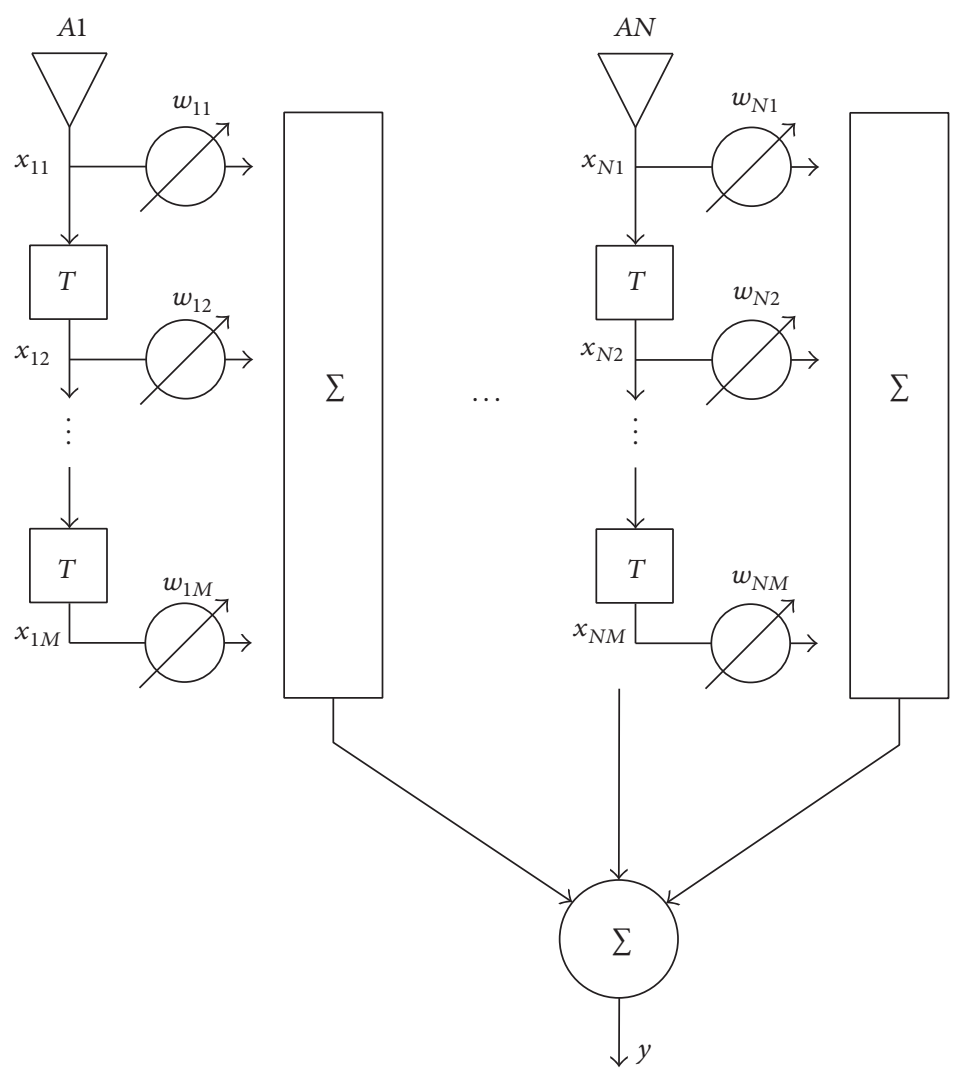

Figure 1: The STAP equipped with $N$ elements and $M$ taps.

The power of signal is about $30 \mathrm{~dB}$ lower than the noise, so the signal can be negligible in calculating the correlation matrix. Thus, the correlation matrix of the received data could be expressed as

$$
\begin{aligned}
\mathbf{R}_{x x}= & E\left[\mathbf{x}(t) \mathbf{x}^{H}(t)\right] \\
= & E\left[\mathbf{j}(t) \mathbf{j}^{H}(t)\right]+E\left[\mathbf{n}(t) \mathbf{n}^{H}(t)\right] \\
= & E\left[a_{j}\left(\omega_{I}, \theta_{j}\right) j(t)\left(a_{j}\left(\omega_{I}, \theta_{j}\right) j(t)\right)^{H}\right] \\
& +E\left[\mathbf{n}(t) \mathbf{n}^{H}(t)\right]
\end{aligned}
$$

$$
E\left[a_{j}\left(\omega, \theta_{j}\right) a_{j}^{H}\left(\omega, \theta_{j}\right)\right]
$$$$
=\zeta\left[\begin{array}{cccc}
\left\|H_{0}\right\|_{2}^{2} & \left\langle H_{0}, H\right\rangle e^{j \pi \sin \theta_{J}} & \left\|H_{0}\right\|_{2}^{2} e^{j \omega_{I} T} & \left\langle H_{0}, H\right\rangle e^{j\left(\omega_{I} T+\pi \sin \theta_{J}\right)} \\
\left\langle H, H_{0}\right\rangle e^{-j \pi \sin \theta_{J}} & \|H\|_{2}^{2} & \left\langle H, H_{0}\right\rangle e^{j\left(\omega_{I} T-j \pi \sin \theta_{J}\right)} & \|H\|_{2}^{2} e^{j \omega_{I} T} \\
\left\|H_{0}\right\|_{2}^{2} e^{-j \omega_{I} T} & \left\langle H_{0}, H\right\rangle e^{-j\left(\omega_{I} T-\pi \sin \theta_{J}\right)} & \left\|H_{0}\right\|_{2}^{2} & \left\langle H_{0}, H\right\rangle e^{j \pi \sin \theta_{J}} \\
\left\langle H, H_{0}\right\rangle e^{-j\left(\omega_{I} T+j \pi \sin \theta_{J}\right)} & \|H\|_{2}^{2} e^{-j \omega_{I} T} & \left\langle H, H_{0}\right\rangle e^{-j \pi \sin \theta_{J}} & \|H\|_{2}^{2}
\end{array}\right],
$$

where $\mathbf{j}(t)$ and $\mathbf{n}(t)$ stand for the interference and noise, separately. $\sigma_{n}^{2}$ and $\sigma_{j}^{2}$ are the power of noise and interference, respectively. $\mathbf{I}$ is a unit matrix, $\theta_{j}$ is the incident direction of interference, and $\omega_{I}$ is the intermediate frequency.

Then,

$$
\zeta=\frac{1}{2 \pi B}
$$

where $\zeta$ is related to the receiver bandwidth which is $B$, and it could be written as 
$\left\|H_{n}\right\|_{2}^{2}$ is the 2-norm of $H_{n}$ and $\left\langle H_{m}, H_{n}\right\rangle$ represents the inner product with $H_{m}$ and $H_{n}$, which could be denoted as

$$
\left\|H_{m}\right\|_{2}=\sqrt{\int_{-\pi B}^{\pi B}\left|H_{m}(\omega)\right|^{2} d \omega}
$$

$\mathbf{R}_{x x}$

$$
=\left[\begin{array}{cc}
\zeta \sigma_{j}^{2}\left\|H_{0}\right\|_{2}^{2}+\sigma_{n}^{2} & \zeta \sigma_{j}^{2}\left\langle H_{0}, H\right\rangle e^{j \pi \sin \theta_{J}} \\
\zeta \sigma_{j}^{2}\left\langle H, H_{0}\right\rangle e^{-j \pi \sin \theta_{J}} & \zeta \sigma_{j}^{2}\|H\|_{2}^{2}+\sigma_{n}^{2} \\
\zeta \sigma_{j}^{2}\left\|H_{0}\right\|_{2}^{2} e^{-j \omega_{I} T} & \zeta \sigma_{j}^{2}\left\langle H_{0}, H\right\rangle e^{-j\left(\omega_{I} T-\pi \sin \theta_{J}\right)} \\
\zeta \sigma_{j}^{2}\left\langle H, H_{0}\right\rangle e^{-j\left(\omega_{I} T+j \pi \sin \theta_{J}\right)} & \zeta \sigma_{j}^{2}\|H\|_{2}^{2} e^{-j \omega_{I} T}
\end{array}\right.
$$

The inverse of correlation matrix could be denoted as

$$
\mathbf{R}_{x x}^{-1}=\frac{1}{\left|\mathbf{R}_{x x}\right|} \mathbf{R}_{x x}^{*}
$$

$$
\left\langle H_{m}, H_{n}\right\rangle=\int_{-\pi B}^{\pi B} H_{m}(\omega) H_{n}^{H}(\omega) d \omega .
$$
as

Consequently, the correlation matrix could be expressed

$$
\left.\begin{array}{cc}
\zeta \sigma_{j}^{2}\left\|H_{0}\right\|_{2}^{2} e^{j \omega_{I} T} & \zeta \sigma_{j}^{2}\left\langle H_{0}, H\right\rangle e^{j\left(\omega_{I} T+\pi \sin \theta_{J}\right)} \\
\zeta \sigma_{j}^{2}\left\langle H, H_{0}\right\rangle e^{j\left(\omega_{I} T-j \pi \sin \theta_{J}\right)} & \zeta \sigma_{j}^{2}\|H\|_{2}^{2} e^{j \omega_{I} T} \\
\zeta \sigma_{j}^{2}\left\|H_{0}\right\|_{2}^{2}+\sigma_{n}^{2} & \zeta \sigma_{j}^{2}\left\langle H_{0}, H\right\rangle e^{j \pi \sin \theta_{J}} \\
\zeta \sigma_{j}^{2}\left\langle H, H_{0}\right\rangle e^{-j \pi \sin \theta_{J}} & \zeta \sigma_{j}^{2}\|H\|_{2}^{2}+\sigma_{n}^{2}
\end{array}\right] .
$$

where $\left|\mathbf{R}_{x x}\right|$ and $\mathbf{R}_{x x}^{*}$ are the determinant and adjoint matrix of $\mathbf{R}_{x x}$, separately.

$\mathbf{R}_{x x}$ is a Hermite matrix, so its determinant is a constant value. We suppose that $v=\left|\mathbf{R}_{x x}\right|$.

$\mathbf{R}_{x x}^{*}$ can be simplified as

$$
\begin{aligned}
& \mathbf{R}_{x x}^{*} \\
& =\sigma_{n}^{4}\left[\begin{array}{cc}
\left(2 \sigma_{j}^{2} \zeta\|H\|_{2}^{2}+\sigma_{j}^{2} \zeta\left\|H_{0}\right\|_{2}^{2}+\sigma_{n}^{2}\right) & -\sigma_{j}^{2} \zeta\left\langle H_{0}, H\right\rangle e^{j \pi \sin \theta_{J}} \\
-\sigma_{j}^{2} \zeta\left\langle H, H_{0}\right\rangle e^{-j \pi \sin \theta_{J}} & \left(\sigma_{j}^{2} \zeta\|H\|_{2}^{2}+2 \sigma_{j}^{2} \zeta\left\|H_{0}\right\|_{2}^{2}+\sigma_{n}^{2}\right) \\
-\sigma_{j}^{2} \zeta\left\|H_{0}\right\|_{2}^{2} e^{-j \omega_{I} T} & -\sigma_{j}^{2} \zeta\left\langle H_{0}, H\right\rangle e^{j\left(\pi \sin \theta_{J}-\omega_{I} T\right)} \\
-\sigma_{j}^{2} \zeta\left\langle H, H_{0}\right\rangle e^{-j\left(\pi \sin \theta_{J}+\omega_{I} T\right)} & -\sigma_{j}^{2} \zeta\|H\|_{2}^{2} e^{-j \omega_{I} T}
\end{array}\right. \\
& \text { Power inversion (PI) is a typical blind criterion to } \\
& \text { suppress interference, which does not require any a priori } \\
& \text { information. It can form nulls in the direction of strong inter- } \\
& \text { ference with superior adaptive performance, and its suitable } \\
& \text { application is in the condition of strong interference and weak } \\
& \text { signal, especially in GNSS receivers [18]. Its constraint can be } \\
& \text { written as } \\
& \text { min } \quad\left\{\mathbf{w}^{H} \mathbf{R}_{x x} \mathbf{w}\right\} \\
& \text { s.t. } \quad \mathbf{w}^{H} b=1,
\end{aligned}
$$

Power inversion (PI) is a typical blind criterion to suppress interference, which does not require any a priori information. It can form nulls in the direction of strong interference with superior adaptive performance, and its suitable application is in the condition of strong interference and weak signal, especially in GNSS receivers [18]. Its constraint can be

where $b=\left[\begin{array}{llll}1 & 0 & \cdots & 0\end{array}\right]^{\mathrm{T}}$ is the constraint vector.

The weights can be further expressed as

$$
\begin{aligned}
\mathbf{w} & =\mu \mathbf{R}_{x x}^{-1} b=\mu \frac{1}{\left|\mathbf{R}_{x x}\right|} \mathbf{R}_{x x}^{*} b \\
& =\frac{\mu}{v}\left[\left(2 \sigma_{j}^{2} \zeta\|H\|_{2}^{2}+\sigma_{j}^{2} \zeta\left\|H_{0}\right\|_{2}^{2}+\sigma_{n}^{2}\right) \sigma_{n}^{4}\right. \\
& -\sigma_{n}^{4} \sigma_{j}^{2} \zeta\left\langle H, H_{0}\right\rangle e^{-j \pi \sin \theta_{J}}-\sigma_{n}^{4} \sigma_{j}^{2} \zeta\left\|H_{0}\right\|_{2}^{2} e^{-j \omega_{I} T} \\
& \left.-\sigma_{n}^{4} \sigma_{j}^{2} \zeta\left\langle H, H_{0}\right\rangle e^{-j\left(\pi \sin \theta_{J}+\omega_{I} T\right)}\right]=\varsigma\left[2 \sigma_{j}^{2} \zeta\|H\|_{2}^{2}\right.
\end{aligned}
$$

The above approximate condition is that the jammer power is much higher than the noise, which is suitable for the actual situation.

The weight vector could be normalized as

$$
\begin{aligned}
\mathbf{w} & =\alpha\left[2\|H\|_{2}^{2}+\left\|H_{0}\right\|_{2}^{2}-\left\langle H, H_{0}\right\rangle e^{-j \pi \sin \theta_{J}}\right. \\
& \left.-\left\|H_{0}\right\|_{2}^{2} e^{-j \omega_{I} T}-\left\langle H, H_{0}\right\rangle e^{-j\left(\pi \sin \theta_{J}+\omega_{I} T\right)}\right],
\end{aligned}
$$

where $\alpha$ is the factor of normalization and it could be denoted as

$$
\alpha=\frac{1}{\sqrt{\left(2\|H\|_{2}^{2}+\left\|H_{0}\right\|_{2}^{2}\right)^{2}+2\left|\left\langle H, H_{0}\right\rangle\right|^{2}+\left\|H_{0}\right\|_{2}^{4}}} .
$$


According to Figure 1, (5), and (8), the array output signal in time domain can be represented as

$$
\begin{aligned}
y(t) & =w^{H} \mathbf{x} \\
& =\sum_{i=1}^{N} \sum_{m=1}^{M} x_{0}(t-(i-1) \tau(\theta)-(m-1) T) w_{i m}^{*},
\end{aligned}
$$

where $x_{0}(t)$ is the sample value of reference array element in the time of $t$. The Fourier transform of (24) can be expressed as

$$
\begin{aligned}
Y(\omega) & =\sum_{i=1}^{N} \sum_{m=1}^{M} X(\omega) e^{-j \pi(i-1) \sin \theta} e^{-j \omega(m-1) T} w_{i m}^{*} \\
& =X(\omega) \sum_{m=1}^{M} e^{-j \omega(m-1) T}\left[\sum_{i=1}^{N} e^{-j \pi(i-1) \sin \theta} w_{i m}^{*}\right] .
\end{aligned}
$$
as

Therefore, the transfer function of STAP can be obtained

$$
H(\omega, \theta)=\sum_{m=1}^{M} e^{-j \omega(m-1) T}\left[\sum_{i=1}^{N} e^{-j \omega(i-1) \tau(\theta)} w_{i m}^{*}\right]
$$

According to the space-time steering vector in (9), the transfer function of STAP in the direction of interference can be expressed as

$$
\begin{aligned}
& H\left(\omega, \theta_{J}\right)=w^{H} \mathbf{a}\left(\omega, \theta_{J}\right)=\alpha\left(2\|H\|_{2}^{2}+\left\|H_{0}\right\|_{2}^{2}\right. \\
& \left.-\left\langle H_{0}, H\right\rangle-\left(\left\|H_{0}\right\|_{2}^{2}+\left\langle H_{0}, H\right\rangle\right) e^{-j\left(\omega-\omega_{I}\right) T}\right) \\
& =\alpha\left(2 \int_{-\pi B}^{\pi B}|A(\omega)|^{2} d \omega+2 \pi B\right. \\
& -\int_{-\pi B}^{\pi B} A(\omega) e^{-j \varphi(\omega)} d \omega \\
& \left.-\left(2 \pi B+\int_{-\pi B}^{\pi B} A(\omega) e^{-j \varphi(\omega)} d \omega\right) e^{-j\left(\omega-\omega_{I}\right) T}\right) .
\end{aligned}
$$

The mismatched steering vector is used in (17), because the received data are all from the mismatched channel. However, the ideal steering vector is used in (27), because the mismatched parameters are unknown when we estimate the transfer function. If the same steering vector is used in (17) and (27), the transfer function would be meaningless.

\section{Impact on Interference Suppression}

If the channel is ideal, (27) could be expressed as

$$
H\left(\omega, \theta_{J}\right)=\alpha\left(4 \pi B-4 \pi B e^{-j\left(\omega-\omega_{I}\right) T}\right) \approx 0 .
$$

Hence, the interference could be suppressed perfectly. However, the channels are always mismatched, and the performance of interference suppression is also limited.

Suppose the interference is the smooth Gaussian white noise, and its bandwidth has covered the entire bandwidth of signal. According to (27), the output power of interference can be expressed as

$$
\begin{aligned}
& P_{J \text {-OUT }}=\frac{P_{J-\text { IN }}}{2 \pi B} \int_{-\pi B}^{\pi B} \mid\left(2 \int_{-\pi B}^{\pi B}|A(\omega)|^{2} d \omega+2 \pi B\right. \\
& -\int_{-\pi B}^{\pi B} A(\omega) e^{-j \varphi(\omega)} d \omega \\
& -\left(2 \pi B+\int_{-\pi B}^{\pi B} A(\omega) e^{-j \varphi(\omega)} d \omega\right) \\
& \left.\cdot e^{-j\left(\omega-\omega_{I}\right) T}\right)\left.\right|^{2} d \omega=\frac{\sigma_{J}^{2} \alpha^{2}}{2 \pi B} \\
& \cdot \int_{-\pi B}^{\pi B} \mid\left(2 \int_{-\pi B}^{\pi B}|A(\omega)|^{2} d \omega+2 \pi B\right. \\
& -\int_{-\pi B}^{\pi B} A(\omega) e^{-j \varphi(\omega)} d \omega \\
& -\left(2 \pi B+\int_{-\pi B}^{\pi B} A(\omega) e^{-j \varphi(\omega)} d \omega\right) \\
& \left.\cdot e^{-j\left(\omega-\omega_{I}\right) T}\right)\left.\right|^{2} d \omega .
\end{aligned}
$$

For convenience, we only analyze the above equation in IF, which is supposed to be suitable, because the antijamming performance in other frequencies is close to the performance in IF. Constantly, the output power of interference could be simplified as

$$
\begin{gathered}
P_{J \text {-OUT }}=\frac{2 \sigma_{J}^{2} \alpha^{2}}{\pi B} \int_{-\pi B}^{\pi B} \mid\left(\int_{-\pi B}^{\pi B}|A(\omega)|^{2} d \omega\right. \\
\left.-\int_{-\pi B}^{\pi B} A(\omega) e^{-j \varphi(\omega)} d \omega\right)\left.\right|^{2} d \omega .
\end{gathered}
$$

Assume that

$$
\begin{aligned}
H_{J}(\omega) & =\left.\left|\int_{-\pi B}^{\pi B}\right| A(\omega)\right|^{2} d \omega-\left.\int_{-\pi B}^{\pi B} A(\omega) e^{-j \varphi(\omega)} d \omega\right|^{2} \\
& =\left|\int_{-\pi B}^{\pi B}\left(|A(\omega)|^{2}-A(\omega) e^{-j \varphi(\omega)}\right) d \omega\right|^{2} \\
& \approx \int_{-\pi B}^{\pi B}\left|\left(|A(\omega)|^{2}-A(\omega) e^{-j \varphi(\omega)}\right)\right|^{2} d \omega .
\end{aligned}
$$

Suppose the noise power is $P_{N}$. The interference cancellation ratio (ICR) can be expressed as

$$
\begin{aligned}
\mathrm{ICR} & =\frac{P_{J-\mathrm{IN}}+P_{N}}{P_{J-\mathrm{OUT}}+P_{N}} \\
& =\frac{\sigma_{J}^{2}+\sigma_{n}^{2}}{\left(2 \sigma_{J}^{2} \alpha^{2} / \pi B\right) \int_{-\pi B}^{\pi B} H_{J}(\omega) d \omega+\sigma_{n}^{2}} .
\end{aligned}
$$


According to (32), the ICR is limited by channel mismatch and its limits could be written as

$$
\mathrm{ICR}_{\lim }=\lim _{\sigma_{J}^{2} \rightarrow \infty} \frac{\pi B}{2 \alpha^{2} \int_{-\pi B}^{\pi B} H_{J}(\omega) d \omega} .
$$

The fundamental principle of interference suppression in antenna arrays is to achieve interference cancellation, and (31) can be further simplified as

$$
H_{J}(\omega)=\int_{-\pi B}^{\pi B}\left(A^{2}(\omega)-2 A(\omega) \cos \varphi(\omega)+1\right) d \omega .
$$

The above equation is the main factor to affect the performance of interference suppression. According to (34), we define a channel mismatch impact factor that can be expressed as

$$
F=H_{J}(\omega)
$$

The smaller the value of the impact factor, the better the performance of interference suppression.

We define another parameter which can also reflect the performance of interference suppression and it can be expressed as

$$
H_{F}(\omega)=A^{2}(\omega)-2 A(\omega) \cos \varphi(\omega)+1 .
$$

According to (3), we can obtain that

$$
\varphi(\omega)=R_{p} \cos \omega R_{p n}+\omega R_{b} \approx \omega R_{b}
$$

where $R_{p}$ and $R_{b}$ are in the same order of magnitude, but $\cos \omega R_{p n}$ is less than 1 and also much less than $\omega$, so the above equation can be approximated.

According to (36) and (37), the main factors to affect the performance of interference suppression are the range of amplitude wave, the number of amplitude waves, and the group delay bias.

In the above analysis, channel 2 is nonideal and its mismatch parameters have not been taken into consideration, and we can recognize they are random. But, in the next plotting, the specific mismatch parameters are necessary, and the model in (3) is used and its parameters are listed in Table 1.

Figure 2 shows the impact factors to $H_{F}(\omega)$ in (36). The curve of reference is with the mismatch in Table 1 . The curves marked with $R_{a}=1 \mathrm{~dB}, R_{a n}=3$ cycle, and $R_{b}=10 \mathrm{~ns}$ stand for resetting $R_{a}, R_{a n}$, and $R_{b}$, respectively, whose reset value is listed in the figure, and the other parameter is the same as Table 1 when the parameter has reset. Figure 2 further presents that the main factors to impact the performance of interference suppression are the range of amplitude wave and the group delay bias.

\section{Simulation Experiment}

GNSS software receiver is used in this simulation. The analysis in this paper concerns the performance of interference suppression, and the PI criterion is adapted, so the navigation signals are unnecessary to be generated. The parameters utilized in simulation experiments are listed in Table 2.

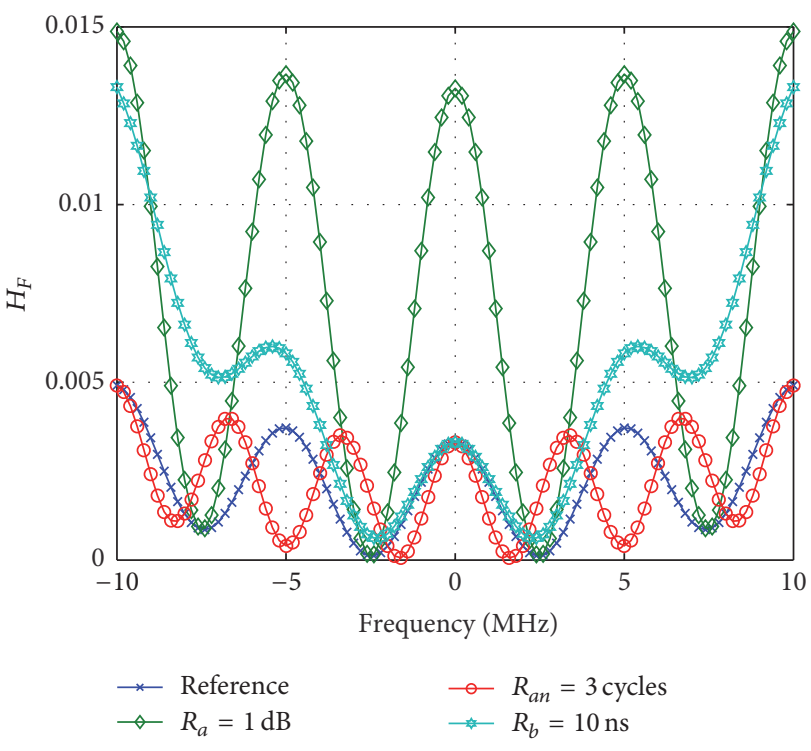

Figure 2: Impact factor to $H_{F}(\omega)$.

TABLE 1: Mismatch parameters.

\begin{tabular}{lc}
\hline Parameters & Value \\
\hline Range of amplitude wave & $0.5 \mathrm{~dB}$ \\
Number of amplitude waves & 2 cycle \\
Range of group delay & $3 \mathrm{~ns}$ \\
Number of group delays & 2 cycle \\
Group delay bias & $4 \mathrm{~ns}$ \\
\hline
\end{tabular}

TABLE 2: Utilized parameters.

\begin{tabular}{lc}
\hline Parameters type & Parameters value \\
\hline Array type & 2-element linear array \\
Interelement spacing & 0.5 wavelength \\
Time-domain taps & 2 \\
Interference type & White Gaussian noise \\
Interference bandwidth & $20 \mathrm{MHz}$ \\
Interference DOA & 15 degrees \\
INR & $35 \mathrm{~dB}$ \\
Sampling frequency & $124 \mathrm{MHz}$ \\
IF & $15 \mathrm{MHz}$ \\
\hline
\end{tabular}

We assume channel 1 is ideal and the characteristic of channel 2 is nonideal whose mismatch parameters are shown in Table 1.

Figure 3 shows the relationship between input INR and ICR. Whether the channel is ideal or mismatch, with the increase of ICR, ICR shows a rising trend. When the channel is ideal, the ICR is approximately equal to the INR and their relationship is linear, and the interference could be completely suppressed. But, in the case of channel mismatch, ICR has different limits in different degree of mismatch. The curve marked with reference shows the ICR in different INR under the channel mismatch whose parameters are set in Table 1 . The curve of $R_{a}=1 \mathrm{~dB}$ expresses the impact of the 


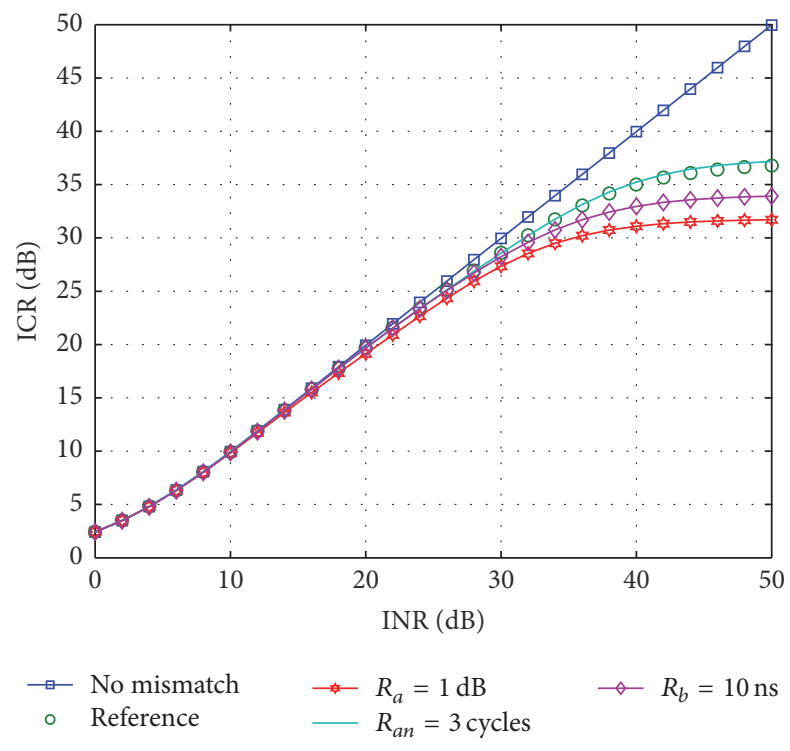

FIGURE 3: ICR in different INR.

range of amplitude wave reset to $1 \mathrm{~dB}$ and the other parameters are the same as reference, whose limit of ICR has decreased. The curve marked with $R_{b}=10 \mathrm{~ns}$ stands for the ICR in different INR under the group delay bias with $10 \mathrm{~ns}$, whose limit of ICR has also been reduced. Nevertheless, when the range of amplitude is reset to 3 cycles, the ICR has little influence compared with the reference.

Figure 4 shows the impact of mismatch extent on ICR. According to the mismatch model in (3), the channel mismatch is related to the range of amplitude wave, the number of amplitude waves, the range of group delay wave, the number of group delay waves, and the group delay bias. In order to put the impact of these parameters on ICR to a diagram, the limits of the parameters are set which are shown in figure, and $x$-axis in figure represents the percentage of those limits. As can be seen from Figure 4, the main influence factors to ICR are the range of amplitude wave and the group delay bias, and the impact of the number of amplitude waves is weaker, and the group delay waves express a little effect on ICR, which is consistent with the conclusion of theoretical analysis.

In (21), (28), (31), (34), and (37), the approximate processing is used. The simulation has proved that the approximate processing is right.

The above simulation is executed only in 2 elements and 2 taps, but these STAP structure is scarce. In the following, we will do simulation with different STAP structure. In all STAP, the distance of the adjacent elements is half wavelength. Regardless of STAP structure, the reference element is ideal, and the other element is nonideal, which would meet the channel mismatch. The degree of channel mismatch is the same as Figure 4. The ICRs in 3 elements' linear array with 2 taps and 4 elements' square array with 2 taps are shown in Figures 5(a) and 5(b). Different elements have different ICRs, but the impact of channel mismatch on ICR is almost the same. The ICRs in 2 elements' linear array with 3 taps and 5 taps are shown in Figures 5(c) and 5(d). The taps are more

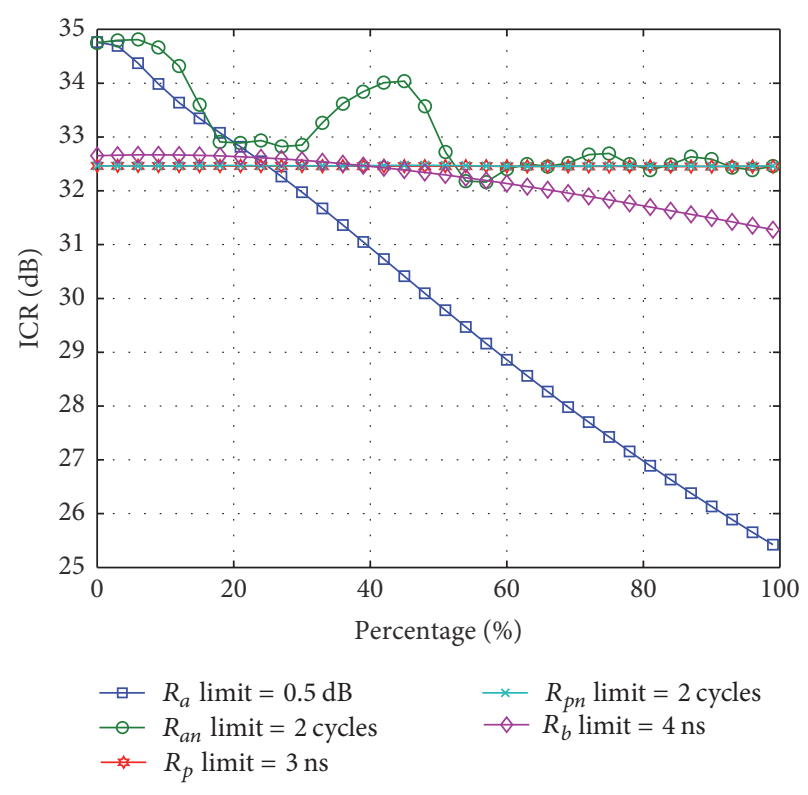

FIGURE 4: ICR in different mismatch degree.

complex on ICR compared to elements. With the increase of taps, the impact of channel mismatch on ICR would become weaker and weaker, because STAP can equalize part of channel mismatch, but its equalization effect is also limited. In addition, the amplitude mismatch is harder to be equalized compared to group delay mismatch.

\section{Conclusion}

In this paper, we have analyzed the impact on antijamming performance of channel mismatch in GNSS antenna arrays receivers. The characteristics of channel mismatch and impact of mismatch level are studied, and the study has been developed through theoretical analysis and simulation experiment.

The outcome of the study is the following:

(1) Under the condition of channel mismatch, the antijamming performance of antenna arrays is limited which is connected to the degree of channel mismatch.

(2) A concept of channel mismatch impact factor is proposed, whose value could directly determine the antijamming performance of antenna arrays.

(3) When the STAP is equipped with 2 elements and 2 taps, the range of amplitude wave and group delay bias are the main factors to impact the antijamming performance, and the number of amplitude waves is next, and the effect of group delay wave is the smallest.

(4) STAP can equalize part of channel mismatch. More taps would bring better equalization effect, and the group delay mismatch is easier to be equalized compared to amplitude mismatch. With the increase of taps, the range and number of amplitude waves have become the main factors to impact the antijamming performance, and the impact of group wave and bias on antijamming performance would become weaker and weaker. 

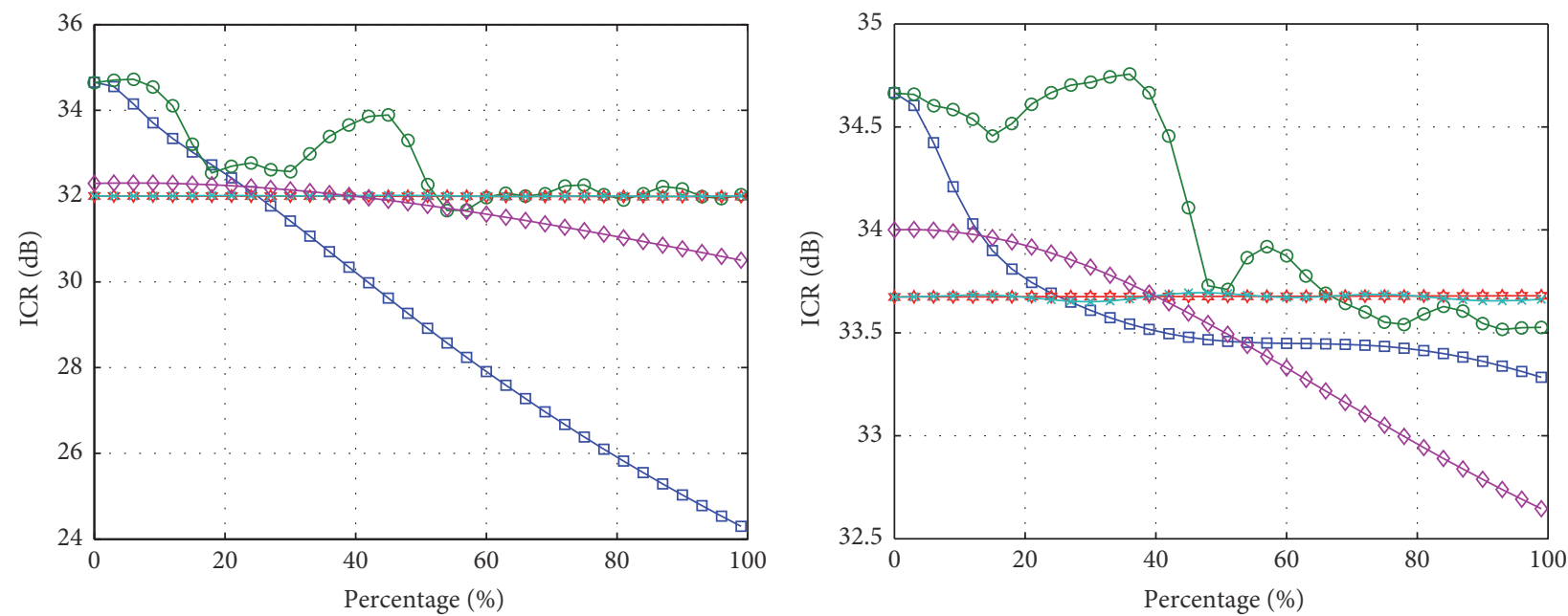

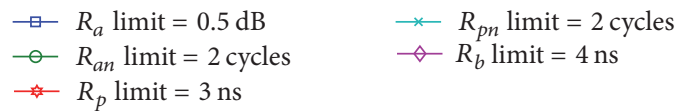

(a) Three elements' uniform linear array with 2 taps

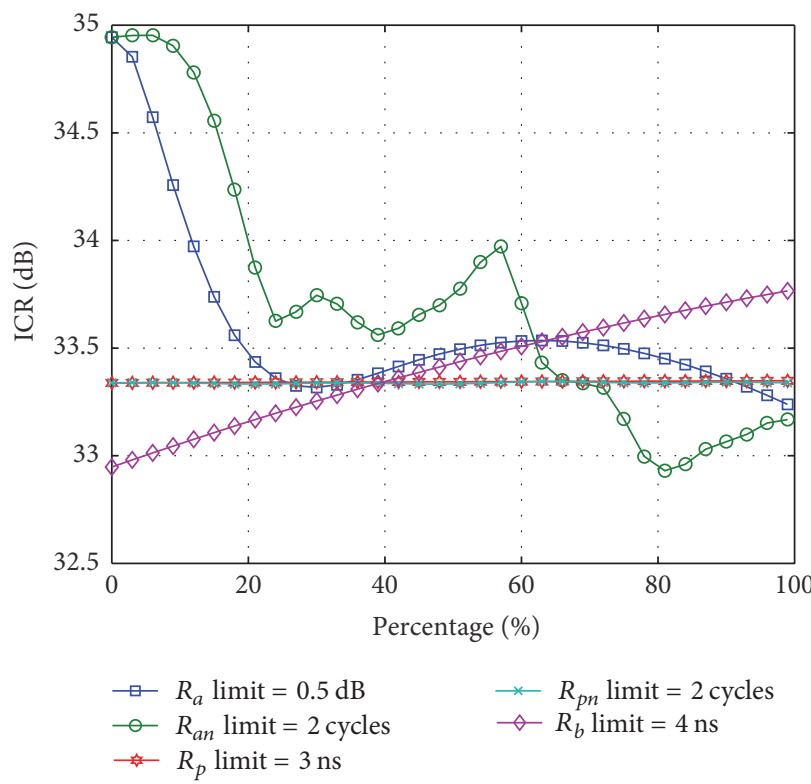

(c) Two elements' uniform linear array with 3 taps

$$
\begin{array}{ll}
\because R_{a} \text { limit }=0.5 \mathrm{~dB} & * R_{p n} \text { limit }=2 \text { cycles } \\
\odot R_{\text {an }} \text { limit }=2 \text { cycles } & \succ R_{b} \text { limit }=4 \mathrm{~ns} \\
\longleftarrow R_{p} \text { limit }=3 \mathrm{~ns} &
\end{array}
$$

(b) Four elements' uniform square array with 2 taps

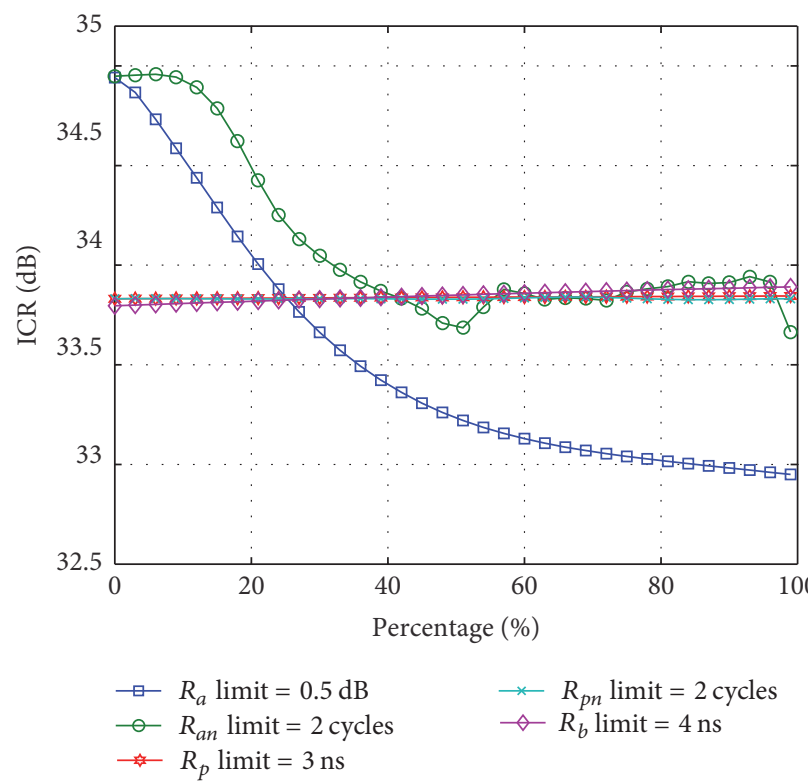

(d) Two elements' uniform linear array with 5 taps

FIGURE 5: ICR in different STAP structure.

Although the effect of group delay wave is the smallest, the group delay wave might affect the measurement in pseudocode and carrier phase seriously, which is the future work for us.

\section{Competing Interests}

The authors declare that they have no competing interests.

\section{References}

[1] N. Kubo, M. Higuchi, T. Takasu, and H. Yamamoto, "Performance evaluation of GNSS-based railway applications," in
Proceedings of the International Association of Institutes of Navigation World Congress (IAIN '15), pp. 1-8, Prague, Czech Republic, October 2015.

[2] W. Alshrafi, U. Engel, and T. Bertuch, "Compact controlled reception pattern antenna for interference mitigation tasks of global navigation satellite system receivers," IET Microwaves, Antennas \& Propagation, vol. 9, no. 6, pp. 593-601, 2015.

[3] G. S. Huang, Z. R. Yi, and Z. C. Zhu, "A novel narrowband interference suppression scheme in GNSS receivers," in Proceedings of the International Conference on Electronics, Communications and Control (ICECC '11), pp. 1252-1256, IEEE, Zhejiang, China, September 2011. 
[4] S. Huo, J. Nie, X. Tang, and F. Wang, "Minimum energy block technique against pulsed and narrowband mixed interferers for single antenna GNSS receivers," IEEE Communications Letters, vol. 19, no. 11, pp. 1933-1936, 2015.

[5] M. G. Amin and W. Sun, "A novel interference suppression scheme for global navigation satellite systems using antenna array," IEEE Journal on Selected Areas in Communications, vol. 23, no. 5, pp. 999-1012, 2005.

[6] S. Wu and Y. Li, "Adaptive channel equalization for space-time adaptive processing," in Proceedings of the IEEE International Radar Conference, pp. 624-628, Alexandria, Va, USA, May 1995.

[7] A. Konovaltsev, M. Cuntz, L. A. Greda, M. V. T. Heckler, and M. Meurer, "Antenna and RF front end calibration in a GNSS array receiver," in Proceedings of the 2010 IEEE International Microwave Workshop Series on ' $R F$ Front-ends for Software Defined and Cognitive Radio Solutions' (IMWS '10), pp. 103-106, February 2010.

[8] W. Ling, Y. Li, S. Xu, Z. Wei, and W. Kun, "Robustness analysis of adaptive anti-jamming algorithms with channel mismatch and mutual coupling for GNSS systems," in Proceedings of the 2nd IEEE International Conference on Signal Processing, Communications and Computing, (ICSPCC '12), pp. 705-710, Hong Kong, China, August 2012.

[9] M. Li, Study on key techniques of digital beamforming in satellite navigation [Ph.D. thesis], Chinese National University of Defense Technology, Changsha, China, 2011.

[10] R. L. Fante and J. J. Vaccaro, "Wideband cancellation of interference in a GPS receive array," IEEE Transactions on Aerospace and Electronic Systems, vol. 36, no. 2, pp. 549-564, 2000.

[11] D. S. De Lorenzo, J. Rife, P. Enge, and D. M. Akos, "Navigation accuracy and interference rejection for an adaptive GPS antenna array," in Proceedings of 19th International Technical Meetings of the Satellite Division of the Institute of Navigation (ION GNSS '06), pp. 763-773, Fort Worth, Tex, USA, September 2006.

[12] F. Gao, Y.-L. Wang, H. Chen, and X.-F. Xue, "Effect of channel mismatch on STAP performance," in Proceedings of the 1st International Congress on Image and Signal Processing (CISP '08), pp. 85-89, IEEE, Hainan, China, May 2008.

[13] K. R. Dandekar, H. Ling, and G. Xu, "Smart antenna array calibration procedure including amplitude and phase mismatch and mutual coupling effects," in Proceedings of the IEEE International Conference on Personal Wireless Communications, pp. 293-297, Hyderabad, India, December 2000.

[14] F. Gao, Y. Wang, H. Chen, and W. Xie, "Effect and equalization results of channel mismatch on STAP performance," Signal Processing, vol. 25, no. 4, pp. 563-565, 2009.

[15] A. J. O'Brien and I. J. Gupta, "Comparison of output SINR and C/N0 for GNSS adaptive antennas," IEEE Transactions on Aerospace and Electronic Systems, vol. 45, no. 4, pp. 1630-1640, 2009.

[16] H. Zhao, Y. Shi, B. Zhang, and M. Shi, "Analysis and simulation of interference suppression for space-time adaptive processing," in Proceedings of the IEEE International Conference on Signal Processing, Communications and Computing (ICSPCC '14), pp. 724-727, IEEE, Guilin, China, August 2014.

[17] Q. Han, J. Nie, S. Huo, and F. Wang, "A high-precision and lowcost planar array signal simulator based on cable delays," IEEE Antennas \& Wireless Propagation Letters, vol. 13, pp. 353-356, 2014.
[18] Z. Ahmad, S. Yaoliang, Q. Du, and I. Ali, "Convergence analysis of null steering antenna utilizing power inversion algorithm," in Proceedings of the 2015 20th IEEE International Seminar/Workshop on Direct and Inverse Problems of Electromagnetic and Acoustic Wave Theory (DIPED '15), pp. 97-101, Lviv, Ukraine, September 2015. 


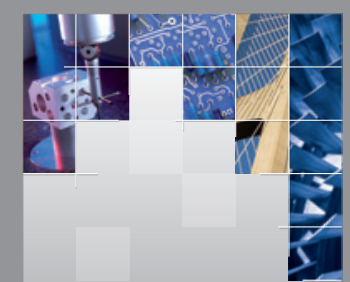

\section{Enfincering}
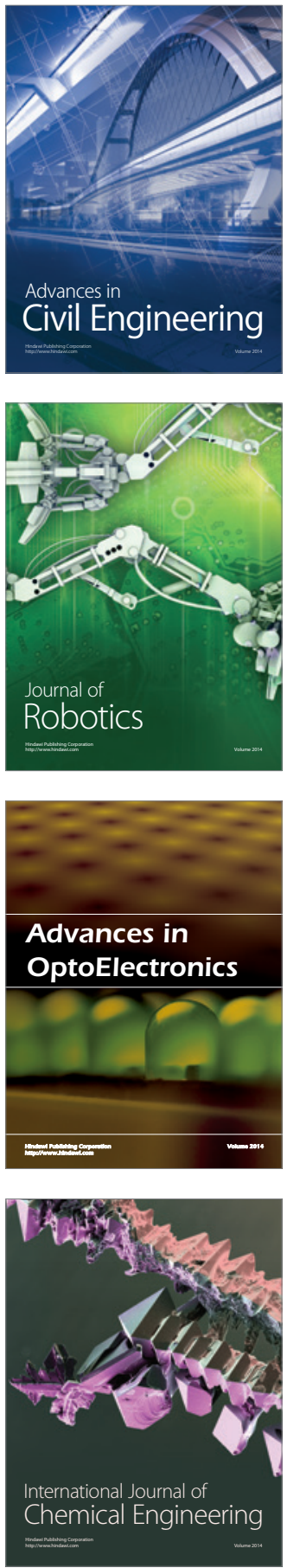

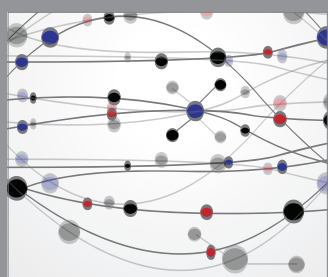

The Scientific World Journal

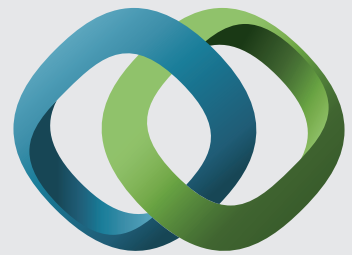

\section{Hindawi}

Submit your manuscripts at

http://www.hindawi.com
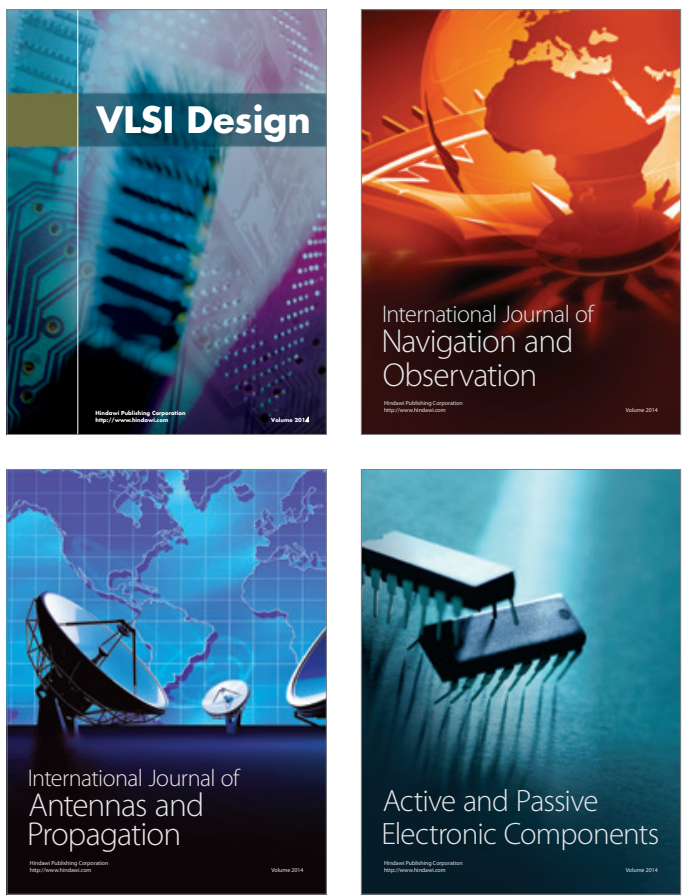
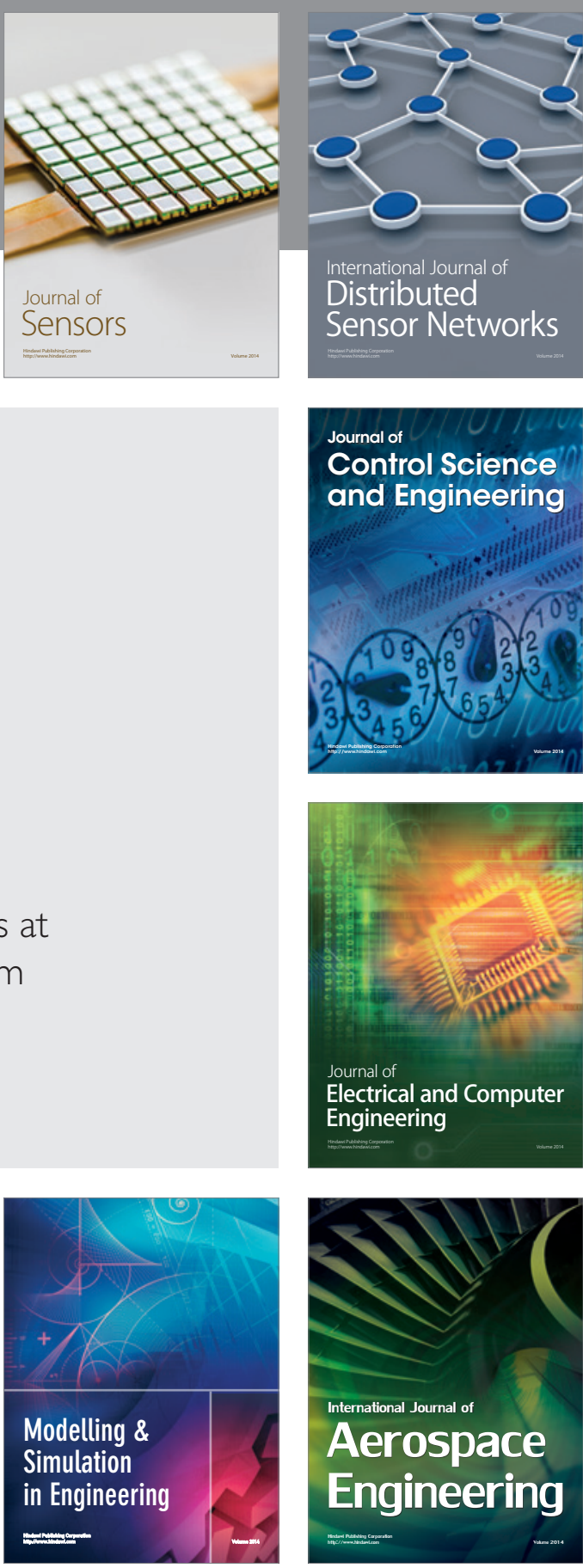

International Journal of

Distributed

Sensor Networks

Journal of

Control Science

and Engineering
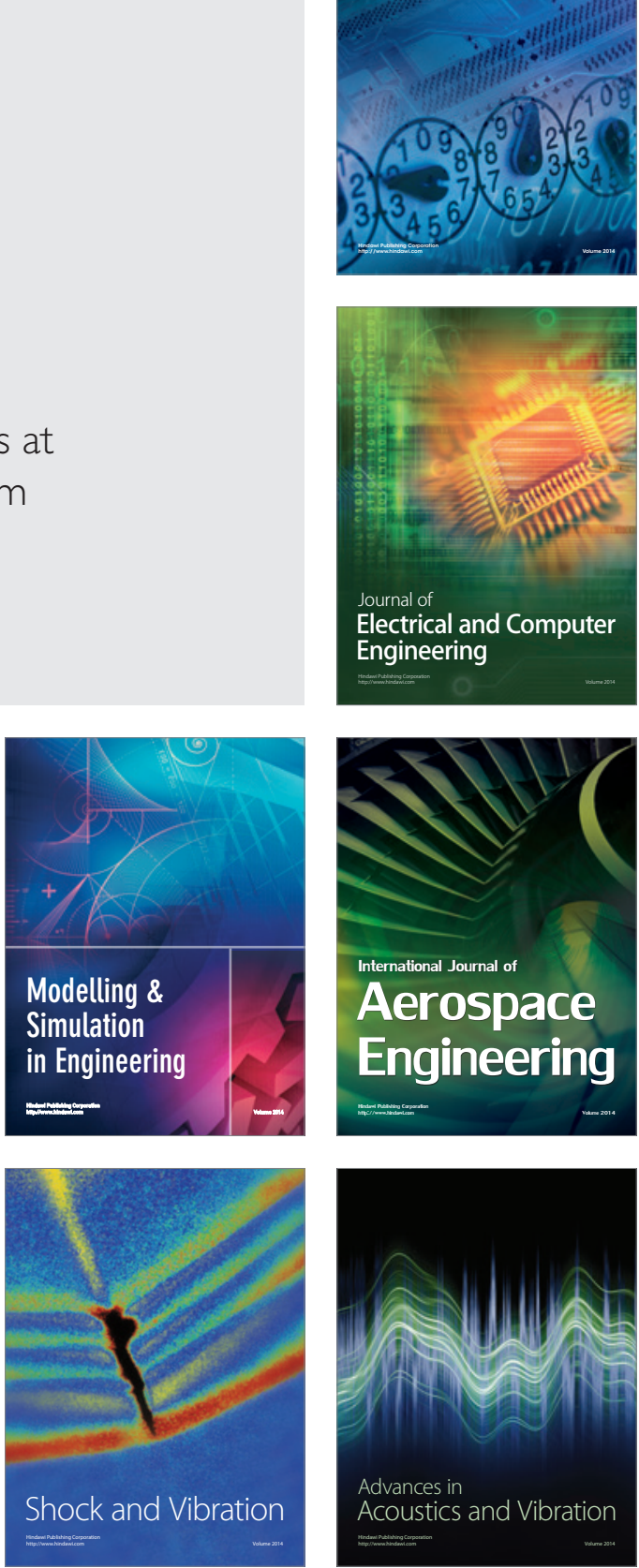